\title{
1 Identification of DIR1-dependant cellular responses required for guard cell systemic
}

\section{2 acquired resistance ${ }^{1}$}

3 Lisa David ${ }^{\mathrm{a}, \mathrm{b}}$, Jianing Kang ${ }^{\mathrm{a}, \mathrm{b}, \mathrm{c}}$, Josh Nicklay ${ }^{\mathrm{d}}$, Craig Dufrense ${ }^{\mathrm{e}}$, and Sixue Chen ${ }^{\mathrm{a}, \mathrm{b}, \mathrm{f}, \mathrm{g}, 2,3}$

$5 \quad{ }^{a}$ Department of Biology, University of Florida, Gainesville, FL 32611, USA

$6{ }^{\mathrm{b}}$ University of Florida Genetics Institute (UFGI), Gainesville, FL 32610, USA

$7 \quad{ }^{\mathrm{c}}$ College of Life Science, Northeast Agricultural University, Harbin 150030, China.

$8{ }^{\mathrm{d}}$ Thermo Fisher Scientific, Somerset, NJ 08873, USA

$9 \quad{ }^{\mathrm{e}}$ Thermo Training Institute, Thermo Fisher Scientific, West Palm Beach, FL 33407, USA

10 f Plant Molecular and Cellular Biology Program, University of Florida, Gainesville, FL 32610, 11 USA

$12{ }^{\mathrm{g}}$ Proteomics and Mass Spectrometry, Interdisciplinary Center for Biotechnology Research 13 (ICBR), University of Florida, Gainesville, FL 32610, USA ORCID IDs: 0000-0003-2529-1863 (L.D.); 0000-0002-0425-8792 (J.K.); 0000-0002-7341-1260 (J.N.); 0000-0003-0785-7798 (C.D.); 0000-0002-6690-7612 (S.C.)

\section{Short title}

19 DIR1 in guard cell systemic acquired resistance.

\section{One-sentence summary}

21 DIR1 affects many biological processes in stomatal guard cells during systemic acquired

22 resistance (SAR), as revealed by multi-omics, and it may function through transporting two $18 \mathrm{C}$

23 fatty acids during SAR.

${ }^{1}$ This material is based upon work supported by the National Science Foundation under Grant No. 1920420.

${ }^{2}$ Author for contact: schen@ufl.edu

${ }^{3}$ Senior author.

The author responsible for distribution of materials integral to the findings presented in this article in accordance with the policy described in the Instructions for Authors (www.plantphysiol.org) is: Sixue Chen (schen@ufl.edu).

S.C., and L.D. conceived and designed the research; L.D., J.K., J.N., and C.D. carried out all experimental work; L.D conducted data analysis, and L.D. and S.C. prepared manuscript. 


\section{ABSTRACT}

26 After localized invasion by bacterial pathogens, systemic acquired resistance (SAR) is induced in

27 uninfected plant tissues, resulting in enhanced defense against a broad range of pathogens.

28 Although SAR requires mobilization of signaling molecules via the plant vasculature, the

29 specific molecular mechanisms remain elusive. The lipid transfer protein-defective in induced

30 resistance 1-1 (DIR1-1) was identified in Arabidopsis thaliana by screening for mutants that

31 were defective in SAR. Here we demonstrate that stomatal response to pathogens is altered in

32 systemic leaves by SAR, and this guard cell SAR defense requires DIR1. Using a multi-omics

33 approach, we have determined potential SAR signaling mechanisms specific for guard cells in

34 systemic leaves by profiling metabolite, lipid, and protein differences between guard cells in

35 wild type and dir1-1 mutant during SAR. We identified two $18 \mathrm{C}$ fatty acids and two $16 \mathrm{C}$ wax

36 esters as putative SAR-related molecules dependent on DIR1. Proteins and metabolites related to

37 amino acid biosynthesis and response to stimulus were also changed in guard cells of dir1-1

38 compared to wild type. Identification of guard cell-specific SAR-related molecules may lead to

39 new avenues of genetic modification/molecular breeding for disease resistant plants.

\section{INTRODUCTION}

Since the dawn of agriculture, epidemics of plant pathogens have caused devastating

43 impacts to food production. The plant bacterial pathogen Pseudomonas syringae (including more

44 than sixty known host-specific pathovars) infect a broad-ranging and agriculturally relevant

${ }^{1}$ This material is based upon work supported by the National Science Foundation under Grant No. 1920420.

${ }^{2}$ Author for contact: schen@ufl.edu

${ }^{3}$ Senior author.

The author responsible for distribution of materials integral to the findings presented in this article in accordance with the policy described in the Instructions for Authors

(www.plantphysiol.org) is: Sixue Chen (schen@ufl.edu).

S.C., and L.D. conceived and designed the research; L.D., J.K., J.N., and C.D. carried out all experimental work; L.D conducted data analysis, and L.D. and S.C. prepared manuscript. 
45 plants (Saint-Vincent et al., 2020). Although it was first isolated from lilac (Syringa vulgaris) in

46 1899, strains of $P$. syringae are found in many important crops, including beans, peas, tomatoes,

47 and rice (Saint-Vincent et al., 2020). P. syringae pv tomato (Pst) is a pervasive phytopathogenic

48 bacterium that causes damage to a wide range of host crop species. It has been a useful model

49 pathogen for studying host immune response since sequencing and annotation of the 6,397,126

50 bp genome and two plasmids was funded by the NSF Plant Genome Research Program (Hirano

51 et al., 2000). Pst infects leaves for chemical nutrients such as carbohydrates, amino acids,

52 organic acids, and ions that are leaked to the leaf apoplast during phloem loading/unloading

53 (Hirano et al., 2000). Pst causes bacterial brown spot disease in fruit and leaves, damaging crop

54 plants. However, more devastating than brown spot is the unique ability of Pst to nucleate

55 supercooled water to form ice. In species of $P$. syringe exhibiting the ice nucleation phenotype,

56 ice-nucleation proteins on the outer membranes of bacterial membranes form aggregates that

57 arrange water into arrays and promote phase change from liquid to solid. The frost-sensitive

58 plants are injured when ice forms in leaf tissues at subzero temperature (Hirano et al., 2000). Pst

59 has been used extensively to study pathogen infection in numerous host plants including tomato

60 and Arabidopsis. The latter is a reference dicot species with a short life-cycle, fully sequenced

61 genome and rich genetic resources, providing an ideal system to understand how plants may be

62 modified to improve their defense and productivity.

63 Systemic Acquired Resistance (SAR) is a long-distance plant immune response that

64 improves immunity of systemic tissues after local exposure to a pathogen (Shah et al., 2013;

65 David et al., 2019). Stomatal pores on leaf surfaces formed by pairs of guard cells are common

66 entry sites for pathogenic bacteria. The specialized guard cells control the opening and closure of

67 stomatal pores in response to environmental conditions (Melotto et al., 2008). When stomatal 
68 guard cells recognize Pst via pattern recognition receptors, stomata close within 1-2 hours and

69 re-open after 3 hours. Re-opening is due to an effector molecule produced by some strains of $P$.

70 syringae called coronatine (COR), which structurally mimics the active form of the plant

71 hormone jasmonic acid-isoleucine (Melotto et al., 2008). As a primary entry site for bacteria into

72 the plant tissue, the stomata are at the frontline in plant immune defense (Zhu et al., 2012). Our

73 previous research showed that systemic leaves of SAR-induced (“primed”) wild type (WT)

74 Arabidopsis have smaller stomata apertures than control plants, and that Pst does not widen

75 stomata aperture in primed leaves, as it does in mock-treated plants (David et al., 2020).

76 Reduced stomatal aperture of primed plants correlated with reduced bacterial entry into leaf

77 apoplastic spaces and reduced bacterial proliferation (David et al., 2020).

78 Using a 3-in-1 extraction method to obtain proteins, metabolites and lipids from the same

79 guard cell samples, we conducted multi-omics to identify SAR-related components in guard cells

80 of WT Arabidopsis and a knockout mutant of Defective in Induced Resistance 1 (DIR1). DIR1

81 encodes a putative apoplastic lipid transfer protein involved in SAR. Arabidopsis plants with

82 mutations in DIR1 exhibit WT-level local resistance to avirulent and virulent Pst, but

83 pathogenesis-related gene expression is abolished in uninoculated distant leaves, and mutants fail

84 to develop SAR (Maldonado et al., 2002). Champigny et al. (2002) examined the presence of

85 DIR1 in petiole exudates from SAR-induced Arabidopsis leaves that were injected with Pst. The

86 exudates from the Pst injected leaves showed the presence of DIR1 beginning at 30 hour-post-

87 infection (hpi) and peaked at $\sim 45$ hpi (Champigny et al., 2002). Interestingly, the small $7 \mathrm{kD}$

88 DIR1 protein was also detected in dimeric form in the petiole exudates (Champigny et al., 2002).

89 DIR1 is conserved in other land plants including tobacco and cucumber, and several identified

90 SAR signals are dependent on DIR1 for long-distance movement, e.g., dehydroabietinal (DA), 
91 azelaic acid (AzA) and glycerol-3-phosphate (G3P) (Adam et al., 2018). Although most of the

92 LTPs have basic pIs, DIR1 has an acidic pI of 4.25. Martinière et al., (2018) found that the

93 apoplastic environment has a more acidic $\mathrm{pH}$ than the cellular environment, ranging between 4.0

94 to 6.3 , so perhaps the acidic pI of DIR1 relates to its function in a more acidic environment

95 where it may be neutral, similar to abscisic acid, which is also transported in the apoplast during

96 stress response (Cornish \& Zeevaart,1985).

97 DIR1 is comprised of 77 amino acids, but despite having cystine residues characteristic

98 with lipid transfer proteins (LTP), it has low sequence identity with the previously characterized

99 LTP1 and LTP2 in Arabidopsis. Lascombe et al. (2008) compared DIR1 to LTP1 by examining

100 their interactions with various lipid substrates, including lysophosphatidyl cholines (LPCs) with

101 various fatty acid chain lengths (LPC C14, LPC C16, and LPC C18). The results showed that

102 DIR1 showed a greater affinity for LPCs with fatty acid chain lengths with $>14$ carbon atoms

103 than LTP1. For the LPC with C18 fatty acid tails, the nonpolar C18 end was completely buried

104 within the barrel structure of the DIR1 protein. DIR1 is unique among the LTPs due to its large

105 internal cavity, capable of carrying two lipid molecules, and a proline-rich PxxPxxP motif

106 (including Proline 24 to Proline 30). The Proline-rich regions of DIR1 may be involved in

107 protein-protein interactions, as these regions are located at the surface of the protein and are fully

108 accessible to the aqueous environment (Lascombe et al., 2008). These regions are putative

109 candidates for docking of a protein signaling partner, or to other cell components. These features

110 may lend themselves well to its role at a SAR-induced LTP because DIR1 is hypothesized to

111 form a complex with azelaic acid included 1 (AZI1) and localize to the endoplasmic reticulum

112 and plasmodesmata (Yu et al., 2013) and to function as a carrier for neutral fatty acids in the

113 apoplast. Many "box-like" LTPs, like DIR1, have a "lid"-like structure that encloses the lipid 
114 ligands inside the hydrophobic cavity during transport in the aqueous environment, and have

115 structural motifs that undergo conformational shifts to allow for lipid loading and unloading

116 (Wong et al., 2019).

117 In this study, a multi-omics approach was employed to identify SAR signaling

118 mechanisms specific for stomatal guard cells. The results show potential involvement of DIR1 in

119 amino acid biosynthesis and carbon metabolism in guard cells during SAR. Importantly, four

120 lipid components with long-chain fatty acids were identified as putative DIR1-related SAR

121 signals in guard cells. The results of guard cell molecules in SAR response have not only led to

122 new insights into the basic function of guard cells in the plant immune response, but also may

123 facilitate biotechnology and marker-based breeding for enhanced crop defense. 


\section{RESULTS}

Altered Stomatal Priming Response in dir1 Correlates with Increased Bacterial Colonization

131 WT Arabidopsis plants improves immunity by allowing fewer bacteria to enter apoplastic spaces

132 (David et al. 2020). In this study, we examined the role of DIR1 in priming of guard cells during

133 SAR using the dir1 knockout mutant and its WT ecotype WS. As previously reported for the

134 Arabidopsis Columbia ecotype (Melotto et al., 2006; David et al. 2021), the basal immune

135 response of the mock-treated WT WS stomata closed after $1 \mathrm{~h}$ exposure to Pst, and then re-

136 opened after $3 \mathrm{~h}$. In contrast, primed WT WS leaves did not exhibit such stomatal immune

137 responses and maintained a small stomatal aperture during the entire period of Pst exposure,

138 similar to that previously observed in the Columbia WT (David et al., 2020) (Figure 1A). There

139 was no significant difference in the stomatal aperture from the primed WS leaves taken at 0,1 ,

140 and $3 \mathrm{~h}$ after Pst exposure (Figure 1B). However, guard cells of systemic leaves of dir1 mutant

141 plants showed an altered response to priming and remain more open at $0 \mathrm{~h}$ and $3 \mathrm{~h}$ compared to

142 WT. It can be noted that due to the perception of pathogen-associated molecular patterns

143 (PAMPs), the $1 \mathrm{~h}$ mock and primed WT and dir1 apertures are similar. Specifically, average

144 stomatal aperture of primed $\operatorname{dir} 1$ leaves was 1.99 vs. $1.67 \mu \mathrm{m}$ in WT at $0 \mathrm{~h}$. At $3 \mathrm{~h}$ it was $2.80 \mathrm{vs}$

$1451.87 \mu \mathrm{m}$ for dir1 and WT, respectively. Interestingly, mock-treated dir1 also showed a larger

146 stomatal aperture at $3 \mathrm{~h}$ after exposure to Pst when compared to mock-treated WT with an

147 average of 3.60 and $2.69 \mu \mathrm{m}$, respectively (Figure 1B).

148 In the dir1 mutant, we found that both the control (mock) and primed dir1 stomatal

149 aperture differed from WT stomata with the same Pst treatments (Figure 1). In control plants

150 (mock), we found that the initial $(0 \mathrm{~h})$ and PAMP response $(1 \mathrm{~h})$ of the dir1 stomata was not 


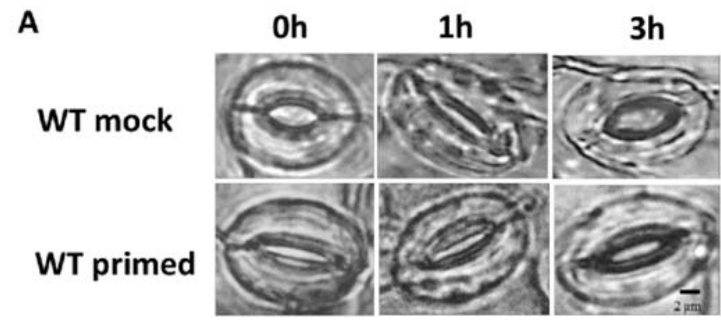

B

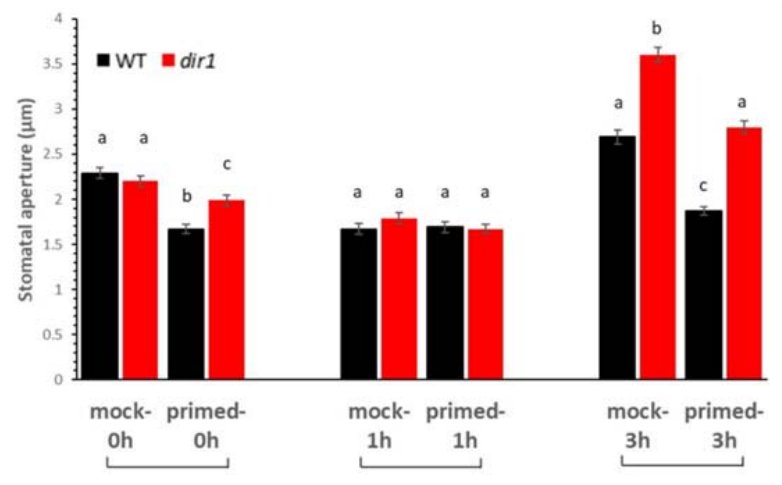

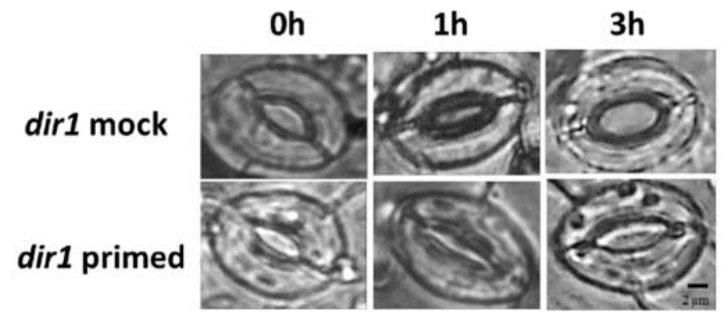

C

D
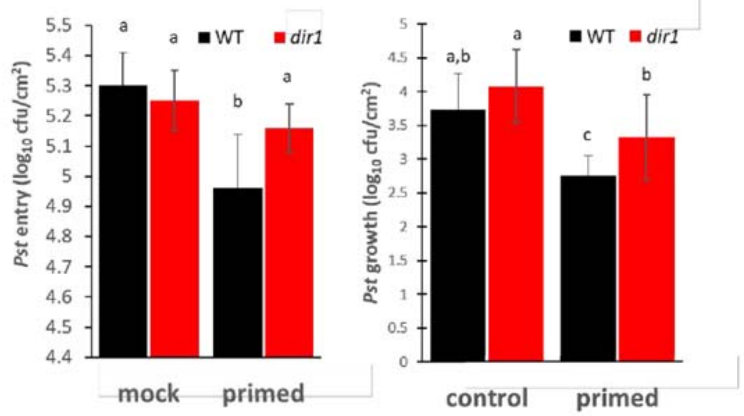

Figure 1. Pathogen entry and growth differences in mock and primed dir1 mutant and wild type (WT) Arabidopsis leaves. A. Images showing representative stomatal apertures in mock and primed $\operatorname{dir} 1$ and WT Arabidopsis leaves after 0,1 , and $3 \mathrm{~h}$ after secondary exposure to Pst DC3000. B. Quantitative measurements of 150 stomata from three replicate experiments. Statistically significant differences were marked by a, b, and c. C. Pst DC 3000 entry results obtained from nine biological replicates of primed and mock dirl and WT plants. The data are presented as average \pm standard error. D. Pst DC 3000 growth results obtained from nine biological replicates of primed and mock plants. The data are presented as average \pm standard error with all p-value $<0.05$. cfu, colony forming unit.

151 statistically different from that of WT stomata to Pst exposure. However, at $3 \mathrm{~h}$ after exposure to

152 Pst, the dir1 mutant displayed a wider stomatal phenotype, indicating that coronatine secreted

153 from Pst had a greater effect on the dir1 stomata than on the WT (Figure 1A and B). The effect

154 of priming on the stomatal aperture of dir1 was also different than that of WT. Intriguingly, the

155 dir1 primed stomata apertures at $0 \mathrm{~h}$ were significantly narrower than the control (mock) dir1

156 stomata, but less narrow than the WT primed stomata. The mock WT and dir1 stomata apertures

157 had no significant difference at $0 \mathrm{~h}$ (2.29 and $2.20 \mu \mathrm{m}$ averages, respectively). After priming,

158 WT stomatal aperture decreased to $1.63 \mu \mathrm{m}$, but the dir1 stomata aperture was reduced to only

$1591.99 \mu \mathrm{m}$, making the primed dir1 stomata apertures significantly different from both the control

160 (mock) dir1 stomata and the primed WT stomata. The $1 \mathrm{~h}$ response to PAMPs from Pst was 
161 similar regardless of genotype (WT vs. dir1) or priming showing the specific response of

162 stomatal closure after PAMP perception. However, at 3 hours post Pst treatment, the dir1 primed

163 stomata phenotype is significantly different from both the dir1 control and the WT primed.

164 Similar to the stomatal phenotype seen at $0 \mathrm{~h}$, the dir1 primed stomata had a narrower aperture

$165(2.8 \mu \mathrm{m})$ than the $\operatorname{dir} 1$ mock $(3.6 \mu \mathrm{m})$ but were less narrow than the WT primed $(1.87 \mu \mathrm{m})$. This

166 demonstrates that although the dir1 mutant appears to be less resistant to the coronatine from the

167 Pst than the WT, it does have improved resistance with priming (Figure 1A and B).

168 Importantly, the altered stomatal phenotype of dir1 directly correlates to Pst entry into

169 the apoplastic spaces of the leaves and reduced stomatal immunity (Figure 1C). There was no

170 significant difference in the number of Pst that were able to enter the apoplast of mock-treated

171 WT, mock-treated dir1, or primed dir1 leaves. Only primed WT stomata were able to reduce Pst

172 entry after $3 \mathrm{~h}$ exposure to the bacterial pathogen (Figure 1C). Although overall immune

173 response of the dir1 mutant is reduced, dir1 plants are still able to mount a SAR response, as

174 demonstrated by the reduced Pst growth after 3 days of exposure in the dir 1 primed leaves

175 (Figure 1D).

176 Pst entry and Pst growth are not significantly different in the mock-treated dir1 vs WT

177 plants, correlating to previous evidence that the dir1 mutant is defective in SAR response, but

178 not in basal pathogen response (Maldonado et al., 2002). In primed leaves, stomata apertures

179 correlate to increased Pst entry into the apoplast of leaves after 3 hours (Figure 1C) and

180 increased growth of Pst after 3 days in the dir1 mutant when compared to WT (Figure 1D). Pst

181 entry is distinctive from Pst growth assays because it involves a more rapid time course (within

182 hours after exposure) as opposed to Pst growth (measured after 3 days). At 3 h the primed WT

183 plants maintain a smaller aperture upon exposure to Pst, while the dir1 plants have larger 
184 stomata apertures. As expected, after $3 \mathrm{~h}$ exposure to Pst, significantly more bacteria entered the

185 apoplasts in the dir1 primed leaves compared to the WT primed leaves (Figure 1C). To examine

186 overall susceptibility to Pst, we measured bacterial growth in the mock and primed systemic

187 leaves. After 3 days of Pst exposure, significantly more bacteria colonized the dir1 primed leaves

188 than the WT primed leaves. (Figure 1D).

\section{Differentially Abundant Proteins in the Primed dir1 and WT Guard Cells}

$190 \quad$ Proteomic analysis of WT versus dir1 primed guard cell samples taken from distal leaves

1913 days after Pst treatment identified 2229 proteins, each with more than one unique peptide (1\%

192 false discovery rate (FDR)). Of the identified proteins, 155 showed differential abundances in the

193 primed WT guard cells compared to the dir1 guard cells, with 25 increased in abundance and

194130 decreased in abundance, by $>2$-fold and a P-value $<0.05$ (Figure 2A). Of the differentially

195 abundant proteins in dir1 primed versus (vs) WT primed, only seven were differentially

196 abundant in dir1mock vs WT mock, indicating that most changes in protein abundance were due

197 to SAR response, rather than to genotype differences.

198 Of the 155 differential proteins, 76 were mapped to the Arabidopsis KEGG pathway.

199 Again, only three of the 76 were differentially abundant in dir1 mock vs WT mock. They were

200 phosphoribosylformylglycinamidine cyclo-ligase (mapped to purine metabolism and

201 biosynthesis of secondary metabolites), vacuolar-sorting protein (in endocytosis pathway), and

202 40S ribosomal protein (in the ribosome pathway). Based on biological functions, the majority of

203 differentially abundant proteins can be broadly categorized into two groups: carbon metabolism-

204 related and amino acid biosynthesis-related. Carbon metabolism-related included 42 proteins

205 from carbon metabolism (13), carbon fixation in photosynthetic organisms (6),

206 glycolysis/gluconeogenesis (6), fructose and mannose metabolism (5), glyoxylate and

207 dicarboxylate metabolism (3), pyruvate metabolism (3), starch and sucrose metabolism (3), and 

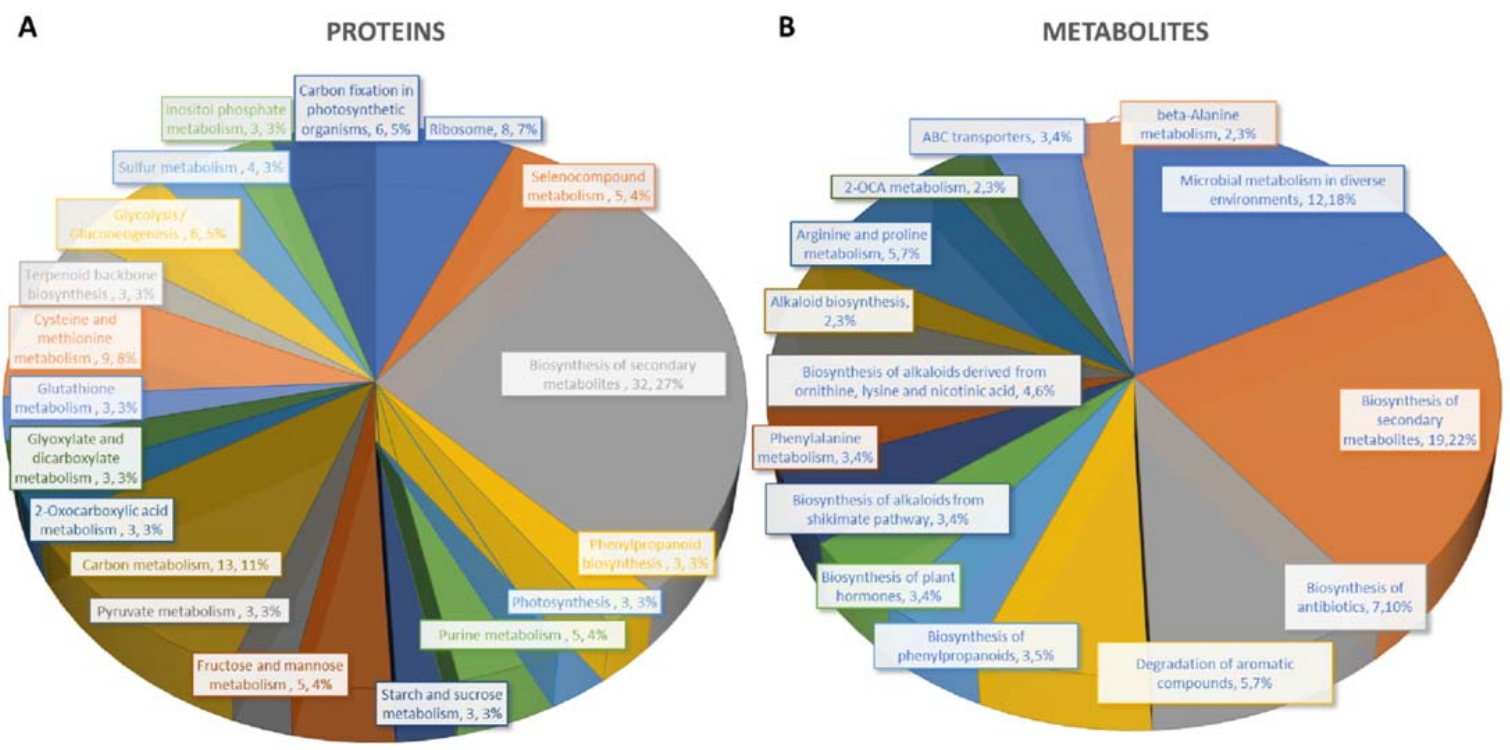

Figure 2. Differential changes of proteins and metabolites in mock and primed dir1 mutant and WT guard cells.

A. Biological functions proteins found in KEGG pathways that are differentially abundant in WT versus dirl primed guard cells.

B. Biological functions metabolites found in KEGG pathways that are differentially abundant in WT versus dirl primed guard cells.

208 photosynthesis (3). Amino acid biosynthesis-related included cysteine and methionine

209 metabolism (9), and purine metabolism (5). Notably, differentially abundant proteins also

210 grouped into inositol phosphate metabolism (3) related to calcium signaling, terpenoid backbone

211 biosynthesis (3) related to sterols and carotenoids, and glutathione metabolism (3) related to

212 redox signaling (Figure 2A).

213 Carbon metabolism-related proteins included fructose-bisphosphate aldolase 3 (FBA3),

214 an enzyme involved in the reversible cleavage of fructose-1,6-bisphosphate into

215 dihydroxyacetone phosphate (DHAP) and glyceraldehyde-3-phosphate (GA3P), and two

216 triosephosphate isomerases (TIM and TPI) that catalyze the reversible isomerization between

217 DHAP and GA3P. There three enzymes exhibited 2-fold decreases in the dir1 primed guard cells

218 compared to WT. Because of the overlap of the carbon metabolism and amino acid biosynthetic

219 KEGG pathways, some differentially abundant proteins were involved in both biological 


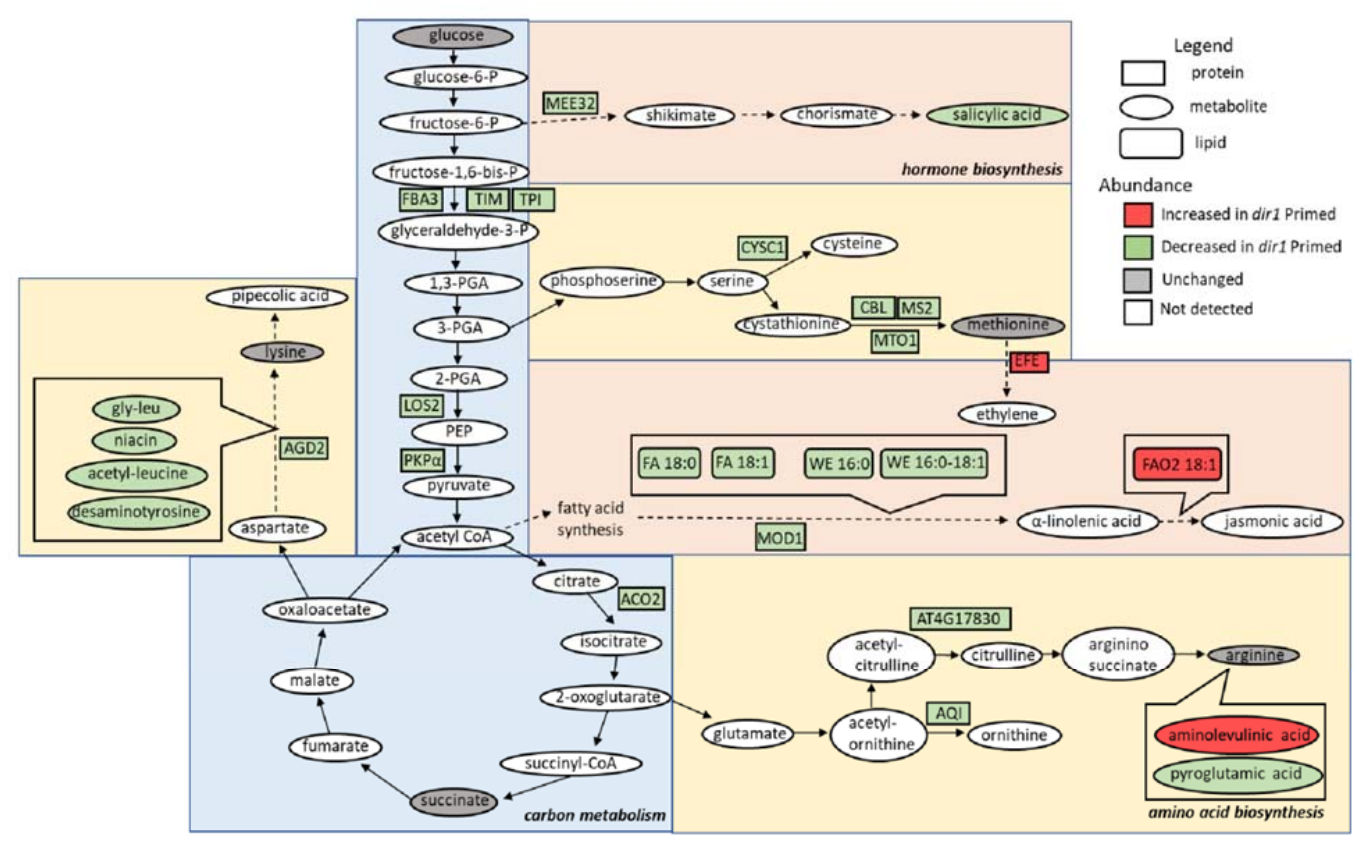

Figure 3. Overview of the role of DIR1 in carbon metabolism, amino acid biosynthesis, and hormone biosynthesis in guard cells during systemic defense response. Loss of DIRl results in altered abundance of proteins, metabolites, and lipids involved in carbon metabolism, amino acid biosynthesis, biosynthesis of plant hormones and secondary metabolites. Proteins that were decreased in dirl guard cells in the carbon metabolism metabolic pathway included: FBA3, TIM, TPI, LOS2, PKP $\alpha$, and ACO2. Proteins that were decreased in dirl guard cells in the amino acid biosynthesis metabolic pathways included: AGD2, CYSC1, CBL, MS2, MTO1, AT4G17830, and AQI, and decreased metabolites in these pathways included: gly-leu, niacin, acetyl-leucine, desaminotyrosine, and pyroglutamic acid. One increased metabolite in dirl guard cells in the arginine biosynthesis pathway was aminolevulinic acid. Proteins that were decreased in dirl guard cells in the biosynthesis of hormones and secondary metabolites metabolic pathways included: MEE32 and MOD1, and decreased metabolites and lipids in these pathways included salicylic acid, stearic acid (FA 18:0), behenic acid (FA 18:1), cetyl oleate (WE 16:0/18:1) and ethyl myristate (WE 16:0). One protein, EFE, and one lipid, FAO2 18:1, were increased in these pathways in the dirl primed guard cells versus WT primed guard cells. Please refer to Supplemental Table 1 for abbreviations.

processes, including a pyruvate kinase family protein (PKP $\alpha$ ) and an enolase (LOS2). Both were

221 decreased more than 2-fold in dir1 primed guard cells compared to WT primed (Figure 3).

224 proteins are also identified in KEGG biosynthesis of secondary metabolites. For example,

225 Maternal Effect Embryo Arrest 32 (MEE32) is a putative dehydroquinate dehydratase and

226 putative shikimate dehydrogenase. It is found in multiple KEGG pathways including:

227 Biosynthesis of amino acids, Metabolic pathways, Phenylalanine, tyrosine and tryptophan

228 biosynthesis, and Biosynthesis of secondary metabolites. Another example is Aconitase 2 
229 (ACO2) which is also found in multiple KEGG pathways, e.g., Biosynthesis of secondary

230 metabolites, Carbon metabolism, 2-Oxocarboxylic acid metabolism, Glyoxylate and

231 dicarboxylate metabolism, Biosynthesis of amino acids, Citrate cycle (TCA cycle), and

232 Metabolic pathways.

233 Amino acid biosynthesis-related proteins included aberrant growth and death 2 (AGD2),

234 which encodes a diaminopimelate aminotransferase involved in disease resistance against Pst

235 and the lysine biosynthesis via diaminopimelate; methionine synthase 2 (MS2), cysteine synthase

$236 \mathrm{C} 1$ (CYSC1) and cystathionine beta-lyase (CBL), which are all involved in cysteine and

237 methionine biosynthesis; and an acetylornithine deacetylase involved in arginine biosynthesis.

238 All mentioned amino acid biosynthesis-related proteins were decreased more than 2-fold in dir1

239 primed guard cells compared to WT primed (Figure 3). Differentially abundant proteins involved

240 in redox pathways included glutathione synthetase 2 (GSH2) and glutathione S-transferase TAU

24120 (GSTU20) related to redox signaling,

242 A pathway enrichment analysis was conducted for the differentially abundant proteins

243 using AGRIGO Singular Enrichment Analysis (SEA) (Supplemental Figures S1 and S2). A

244 graphical representation of GO hieratical groups with all statistically significant terms classified

245 levels of enrichment with corresponding colors. The functional enrichment was found in three

246 general groups including response to stimulus, amino acid metabolic processes, and carbohydrate

247 metabolic processes (Supplemental Figure S1). AGRIGO singular enrichment analysis for

248 cellular components revealed enrichment in intracellular organelles including intracellular

249 membrane bounded organelles, plastids, and chloroplast stroma (Supplemental Figure S2). 


\section{Differential metabolites in the primed dir1 and WT guard cells}

A total of 728 metabolites were identified, and 55 metabolites showed significant

253 changes after the priming treatment in the dir1 versus WT guard cells, with 16 increased and 39

254 decreased in abundance, by $>2$-fold and a P-value $<0.05$ (Figure $2 \mathrm{~B}$ ). Of these differential

255 metabolites, 34 were mapped to KEGG pathways. When grouping by biological function, the

256 largest group of differentially abundant metabolites found in KEGG pathways were related to

257 biosynthesis of secondary metabolites (19) (Figure 2B).

Several differential metabolites are involved in amino acid biosynthesis and hormone

259 metabolism. For example, SA was decreased by more than 4-fold in the dir1 primed guard cells

260 compared to WT samples (Figure 3). However, it should be noted that in dir1 mock versus WT

261 mock the same ratio of decreased SA abundance exits. Metabolites involved in lysine

262 biosynthesis were decreased more than 2-fold in the dir1 primed guard cells compared to WT.

263 They included gly-leu, niacin, acetyl-leucine, and desaminotyrosine. Metabolites involved in

264 arginine biosynthesis were also changed. For example, pyroglutamic acid that decreased more

265 than 2-fold, and aminolevulinic acid increased more than 4-fold in the dir1 primed guard cells

266 compared to WT guard cells. Malic acid, which is related to carbon metabolism, was increased

267 1.8-fold in dir1 versus WT primed guard cells, but was decreased by nearly 2-fold in dir1 vs WT

268 mock. When malic acid in the guard cell is pumped out to the apoplast, water moves out

269 reducing turgor pressure in the guard cells and closing the stomata (Santelia and Lawson, 2016).

\section{Differential Lipids in the Primed dir1 and WT Guard Cells}

272 A total of 1197 lipids were identified, and 88 lipids showed significant changes in guard

273 cells after the priming of the dir1 vs WT guard cells (with 37 increased and 49 decreased by $>2$ -

274 fold). Of the differential lipids, 15 were mapped to KEGG pathways and their biological 


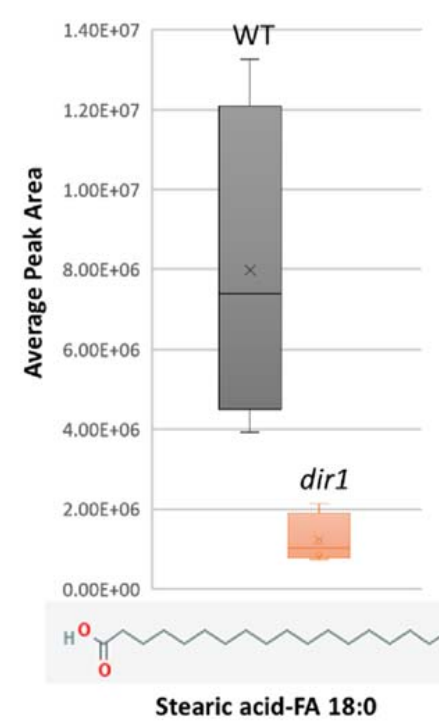

Stearic acid-FA 18:0

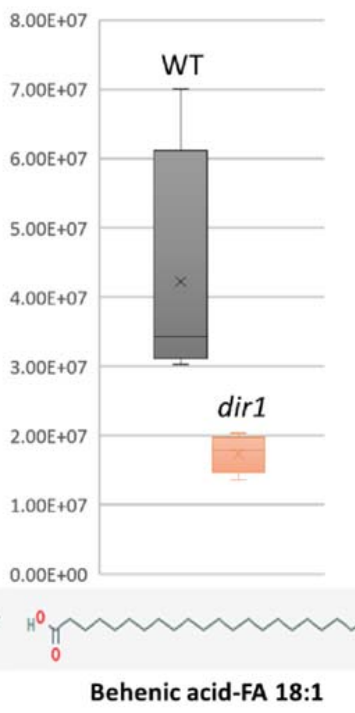

Behenic acid-FA 18:1

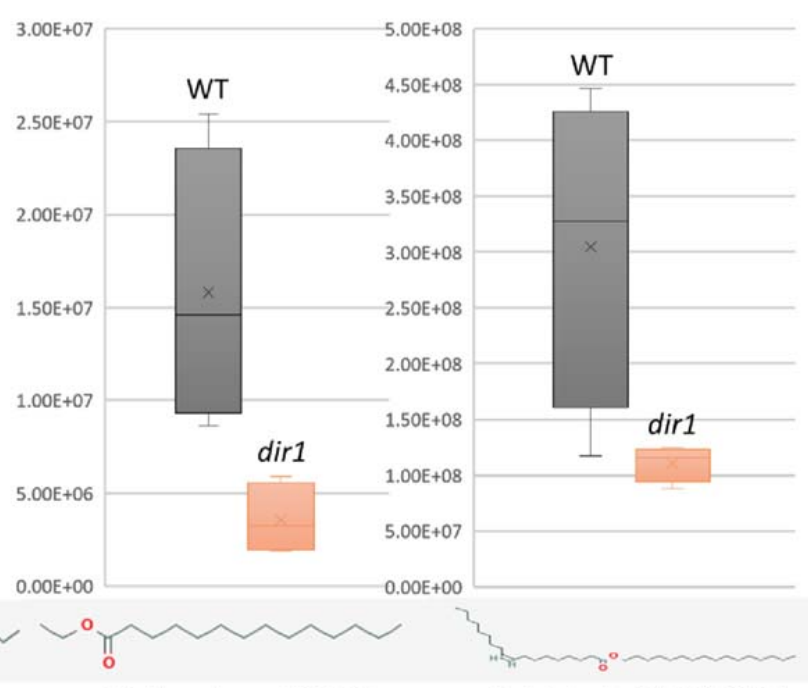

Ethyl myristate-WE 16:0

Cetyl oleate-WE 16:0/18:1

Figure 4. Differentially abundant lipids identified in $\operatorname{dir} 1$ and WT guard cells. A. Bar graphs showing decreases of two longchain fatty acids, stearic acid (FA 18:0) and behenic acid (FA 18:1) and two wax esters, cetyl oleate (WE 16:0/18:1) and ethyl myristate (WE 16:0) decreased > 2-fold in the dirl versus WT guard cells. Chemical structures of stearic acid (FA 18:0), behenic acid (FA 18:1), cetyl oleate (WE 16:0/18:1) and ethyl myristate (WE 16:0) are shown. The error bar represents standard deviation of the mean value.

275 functions largely fell into two categories: biosynthesis of fatty acids and biosynthesis of

276 secondary metabolites. Notably these lipids included FAO2 18:1, isoleukotoxin diol (DiHOME)

277 involved in linoleic acid metabolism (a precursor for jasmonic acid). It was increased 2.1-fold in

278 the dir1 vs WT primed guard cells. We also found two long-chain fatty acids (FA) including

279 stearic acid (FA 18:0) and behenic acid (FA 18:1) and two wax esters (WE) including cetyl

280 oleate (WE 16:0/18:1) and ethyl myristate (WE 16:0). They were all decreased more than 2-fold

281 in the dir1 vs WT guard cells (Figure 3, Figure 4). Ethyl myristate is a long-chain fatty acid ethyl

282 ester resulting from the condensation of the carboxy group of myristic acid with

283 the hydroxy group of ethanol. Palmityl oleate is a wax ester obtained by the condensation of

284 hexadecan-1-ol with oleic acid. Interestingly, both stearic acid and behenic acid were not

285 significantly changed in the dir1 mock vs WT mock, indicating that this change in FA amount is 
286 due to priming, further supporting that they may be the 18C lipid signals potentially transported

287 by DIR1. As to the two wax esters (cetyl oleate and ethyl myristate), they were already more

288 than 2-fold reduced in dir1 mock vs WT mock, indicating genotypic difference rather than

289 priming effect.

291 DIR1 localization and protein interactions with DIR1

292 Using the Interaction Viewer at the Bio-Analytic Resource for Plant Biology (BAR)

293 (bar.utoronto.ca/eplant), localizations of DIR1 and proteins that interact with DIR1

294 (AT5G48485) were determined (Figure 5A). Cellular localizations of DIR1 included

295 peroxisomes, Golgi apparatus, endoplasmic reticulum, and plasma membrane. Protein-protein

296 interactions that have been experimentally determined, indicated by the straight, green lines,

297 occur between DIR1 and both ubiquitin-like protein (AT1G68185) and chitin elicitor receptor

298 kinase 1 (CERK1, AT3G21630). Based on Araport 11 annotation, CERK1 is a LysM receptor-

299 like kinase, and has a typical RD signaling domain in its catalytic loop and possesses

300 autophosphorylation activity. GO biological functions of CERK1 include perception and

301 transduction of the chitin oligosaccharide elicitor in innate immune response to fungal

302 pathogens. CERK1 is located in the plasma membrane and cytoplasm and phosphorylates LIK1,

303 an LLR-RLK that is involved in innate immunity (Rebaque et al, 2021; Junková et al, 2021).

304 However, neither the ubiquitin-like protein nor CERK1 were identified in our proteomics results 305 (Supplemental Table S1).

306 The GeneMANIA tool at the BAR resource was used to predict other genes/gene

307 products associated with DIR1. Predicted, co-expression, and genetic interaction networks found 308 associated genes/gene products (Figure 5B). In addition to DIR1, our proteomics identified

309 several lipid transfer proteins including LTP1, LTP5, LTP6, Plastocyanin (PETE1) and LTPG6 

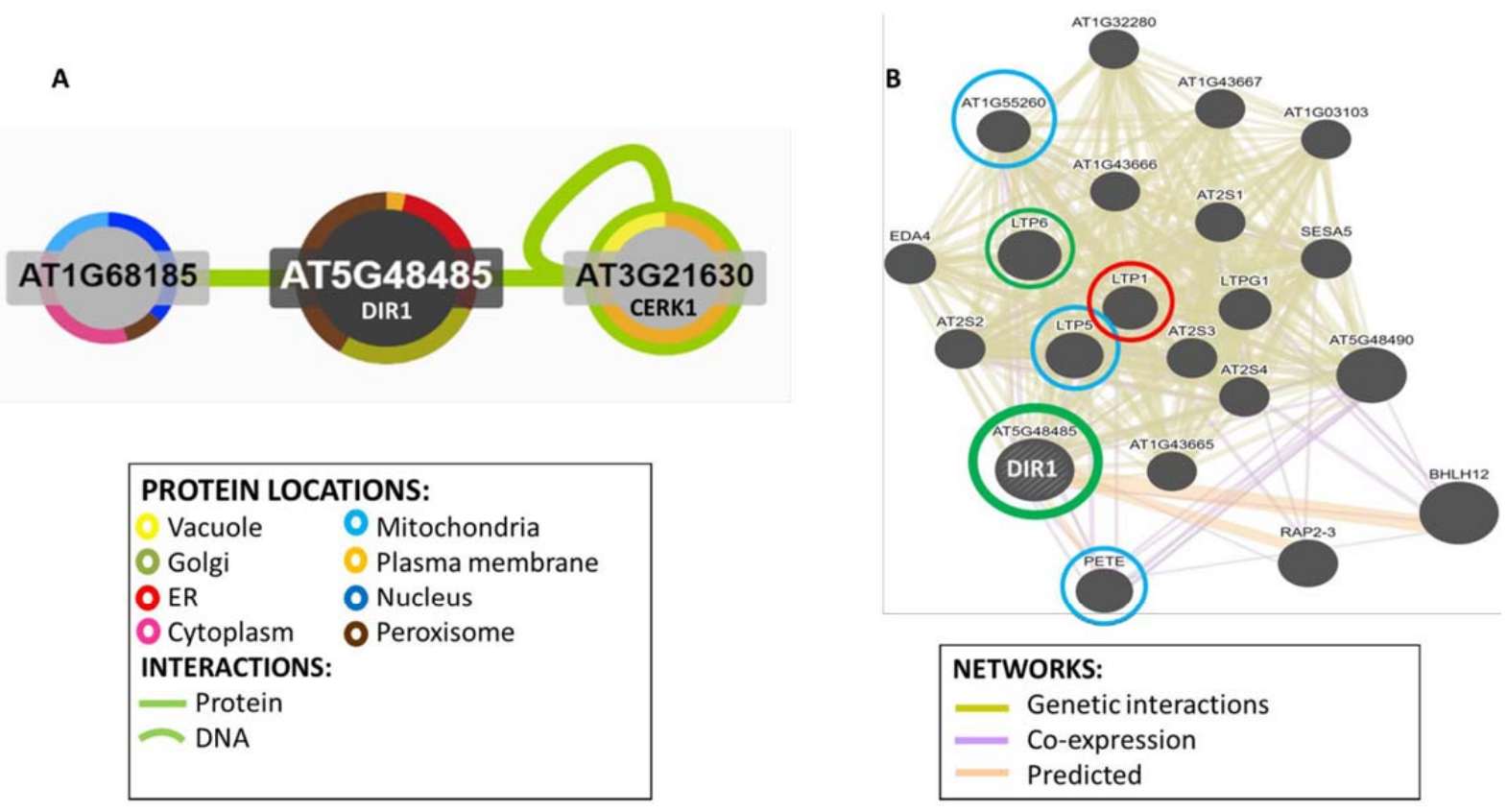

Figure 5. Identification of potential interacting proteins with DIR1. A. Protein interaction image was generated using Interaction Viewer at bar.utoronto.ca/eplant. Border color indicates protein location. Green lines indicate protein and DNA interactions that have been experimentally determined. B. GeneMANIA tool from bar.utoronto.ca/eplant was used to predict other genes/gene products associated with DIR1 (AT5G48485). Predicted, co-expression, and genetic interaction networks found associated genes/gene products. Proteins identified in guard cell samples are circled. Circle colors indicate increased (red), decreased (green), or unchanged (blue) proteins in $\operatorname{dirl}$ versus WT primed guard cells.

310 (AT1G55260) from guard cell samples. LTPG6 is a glycosylphosphatidylinositol-anchored lipid

311 transfer protein involved in defense response to fungus. LTP1 (AT2G38540), is a non-specific

312 lipid transfer protein that binds calmodulin in a $\mathrm{Ca}^{2+}$-independent manner. LTP1 is specifically

313 expressed in the L1 epidermal layer and is localized to the cell wall (Fahlberg et al., 2019).

314 LTP1, LTP5 (AT3G51600) and LTP6 (AT3G08770) are predicted to encode pathogenesis-

315 related (PR) proteins and are members of the PR-14 protein family (Sels et al., 2008). The

316 mRNA of LTP1 is cell-to-cell mobile (Bogdanov et al., 2016). PETE1 is one of two Arabidopsis

317 plastocyanins (PETE1 and PETE2). Its mRNA expression is one-tenth of the level of PETE2.

318 Although PETE2 is involved in copper homeostasis, PETE1 is not responsive to increased

319 copper levels, but it may participate in electron transport during copper-limiting conditions 
320 (Abdel-Ghany, 2009; Weigel et al., 2003). DIR1 was not present in our dir1 knockout mutant

321 samples, and LTP6 was significantly decreased in the dir1 versus WT after priming. LTP1 was

322 increased in dir1 versus WT, and LTP5, PETE and LTPG6 were unchanged during priming in

323 dir1 versus WT guard cells (Figure 5B). DIR1 was associated with LTP1, LTP5, LTP6 and

324 LTPG6 via genetic interaction networks, and with PETE1 via predicted and co-expression

325 networks (Figure 5B).

326

327 


\section{DISCUSSION}

\section{9 dir1 is Deficient in Both Local and Systemic Guard Cell Immune Responses}

Although SAR has largely been studied at the level of leaf or whole plant level, we have

331 recently shown evidence that SAR affects guard cell response to the bacterial pathogen Pst

332 (David et al., 2020). DIR1 is required for movement of several chemically diverse SAR signals

333 including DA, G3P, AzA, and possibly MeSA (Adam et al., 2018). As we have recently reported

334 stomatal movement and guard cell molecular changes underlying stomatal SAR responses

335 (David et al., 2020), here we first characterized the stomatal movement phenotype of the dir1

336 mutant versus WT in response to Pst. Results from our work and previous studies (Melotto et al.,

337 2008; Pang et al., 2020) clearly showed that stomatal guard cells from different genotypes of

338 Arabidopsis (WS and Columbia) exhibited similar basal immune responses. After priming for

339 three days, stomata from the WT WS leaves had an initial narrow aperture compared to control

340 (mock) stomata, and they maintain this narrow aperture during PAMP perceptions at 1 hour and

341 also at 3 hours after Pst treatment. This result is also similar to the Columbia WT plants (David

342 et al., 2020),

343 In the dir1 mutant, at $3 \mathrm{~h}$ after exposure to Pst the dir1 mutant displayed a larger stomatal

344 aperture, indicating that coronatine secreted from Pst had a greater effect on the dir1 guard cells

345 than on the WT. The effect of priming on the dir1 stomata was also different from the WT

346 stomata. The dir1 primed stomata apertures at $0 \mathrm{~h}$ were narrower than the mock dir1, but less

347 narrow than the WT primed stomata. At $3 \mathrm{~h}$ post Pst treatment, the dir1 primed stomatal aperture

348 is smaller than mock-treated, but less narrow than the WT primed. The altered stomatal aperture

349 of dir1 directly correlates to Pst entry into the apoplastic space (Figure 1). Clearly, although the

350 dir1 mutant appears to be less resistant to the coronatine than the WT, it does have improved

351 resistance after priming. This result is consistent with previous literature, which showed a partial 
352 SAR-competent phenotype of dir1 (Champigny et al., 2013). Although the partial SAR-

353 competent phenotype of dir1 was able to reduce the Pst growth, it did not decrease the entry of

354 Pst via the stomatal pores. Therefore, dir1 is deficient in both local and systemic guard cell

355 immunity.

\section{DIR1 affects Guard Cell Carbon Metabolism and Amino Acid Biosynthesis During SAR}

Based on our multi-omic results, the majority of the differential proteins and metabolites

358 (including lipids) were in the carbon metabolism, amino acid biosynthesis and secondary

359 metabolite biosynthesis pathways (Figures 2 and 4). Most of the molecules were lower in the

360 primed dir1 guard cells than the primed WT guard cells. These results indicate that DIR1-

361 dependent SAR is necessary for regulation of amino acid biosynthesis and secondary metabolites

362 in guard cells. It also indicates that guard cells attenuate their carbon metabolic pathways to

363 divert resources to amino acid biosynthesis in response to priming in WT, and that this process is

364 at least partially dependent on DIR1 in guard cells. In addition, the differential proteins enriched

365 for plastid and chloroplast components again support alterations in carbon metabolic pathways

366 induced by SAR.

367 One interesting aspect of our results is that we did not identify changes in pathogenesis-

368 related (PR) proteins in the dir1 primed guard cells. Similarly, the abundance of AzA was not

369 significantly different in the primed dir1 versus WT guard cells. On the other hand, the key

370 regulatory SAR metabolite SA showed a 50-fold decrease in the primed dir1 vs WT guard cells.

371 Previously, we reported that primed guard cells in uninoculated leaves of Arabidopsis narrowed

372 stomatal apertures, reduced entry of Pst into the leaves, and had increased SA in primed guard

373 cells compared to mock guard cells (David et al., 2020). The lower SA in the primed dir1 guard

374 cells correlates well with our previous findings and demonstrates that DIR1 is required to

375 transmit the long-distance SAR signal to the guard cells in uninfected leaves and increase SA in 
376 the primed guard cells. Recently, translocation of SA from primary infected tissue to distal

377 uninfected leaves was shown to likely occur via the apoplastic space between the cell wall and

378 plasma membrane (Lim et al., 2016). Unlike the SAR-induced signals G3P and AzA, which

379 were preferentially transported via symplastic transport and through plasmodesmata, pathogen

380 infection resulted in increased SA accumulation in the apoplastic compartment, and SAR-

381 induced accumulation was unaffected by defects in symplastic transport via plasmodesmata (Lim

382 et al., 2016). Mature guard cells have callose depositions that block plasmodesmata, and thus

383 SAR-signals that can be transported via the apoplast, rather than the symplast, would logically be

384 able to affect the guard cells during SAR. Alternatively, SA could be de novo synthesized in the

385 primed guard cells. This SA biosynthesis is also affected by DIR1 mutation. How DIR1 regulates

386 SA biosynthesis is not known.

\section{Lipidomics Revealed Four Long-Chain Fatty Acids Associated with DIR1}

Previously, we found that fatty acids were increased in the primed WT guard cells (David

390 et al., 2020). Here we compared the levels of lipids found in primed WT guard cells to those in

391 the dir1 mutant. Our goal was to identify lipids in guard cells that are dependent on DIR1 during

392 priming. DIR1 has been characterized as a lipid transfer protein, and the core of its structure

393 forms a left-handed super helical arrangement of four $\alpha$-helices building the hydrophobic central

394 cavity. Lascombe et al. (2008) demonstrated that DIR1 showed a greater affinity for LPCs with

395 fatty acid chain lengths with $>14$ carbon atoms and that nonpolar C18 fatty acid tails were

396 completely buried within the barrel structure of the DIR1 protein, presumably allowing non-

397 polar fatty acids to be transported in polar cellular environments. The two long-chain fatty acids

398 (stearic acid (FA 18:0) and behenic acid (FA 18:1)) and two wax esters (cetyl oleate (WE

399 16:0/18:1) and ethyl myristate (WE 16:0)) were all decreased $>2$-fold in the dir1 guard cells 
400 compared to WT guard cells (Figures 3 and 4). As both stearic acid and behenic acid were not

401 significantly changed in dir1 mock vs WT mock, this change in FA levels is likely due to

402 priming, further supporting that they may be the 18C lipid signals transported by the DIR1.

403 Further analysis is required to determine the relationship between DIR1 and these 18C fatty

404 acids. It is reasonable to propose that DIR1 may transfer stearic and behenic acid to guard cells

405 during SAR. Previously we identified an increase in palmitic acid and its derivative 9-

406 (palmitoyloxy) octadecanoic acid in primed WT guard cells and proposed that fatty acids could

407 allow for the development of lipid rafts or other alterations of membrane structure in guard cells,

408 modulating stomatal immune responses (David et al., 2020).

Plant wax esters are neutral lipids with long-chain $\left(\mathrm{C}_{16}\right.$ and $\left.\mathrm{C}_{18}\right)$ or very-long-chain

$410 \quad\left(\mathrm{C}_{20}\right.$ and longer $)$ carbon structures and are mostly found in cuticles where they provide a

411 hydrophobic coating to shoot surfaces (Li et al., 2008). Recently the cuticle has been shown to

412 regulate transport of SA from pathogen-infected to uninfected parts of the plant via the apoplast

413 during SAR (Lim et al., 2020). Lim et al. (2020) found that cuticle-defective mutants with

414 increased transpiration and larger stomatal apertures reduced apoplastic transport of SA and

415 caused defective SAR response. It is interesting to note that our results demonstrate that WT

416 stomata maintain narrow stomata apertures after priming, potentially to reduce transpiration and

417 increase water potential, and possibly routing SA to the apoplast. The dir1 mutant, on the other

418 hand, had larger stomatal apertures, perhaps resulting in defect in SA movement in the apoplast.

419 It is not known whether the mutant has defect in cuticle structure due to the decreases of wax

420 esters (cetyl oleate and ethyl myristate). However, since the decreased cetyl oleate and ethyl

421 myristate in dir1 guard cells after priming were already $>2$-fold reduced in dir1 mock vs WT

422 mock, this was a genotypic difference, rather than a result of priming. If, as reported by Lim et 
423 al. (2020), defects in the cuticle reduce transport of SA, the reduced wax esters in dir1 vs WT

424 could explain the reduce SA in dir1 guard cells (both mock and primed) and contribute to the

425 SAR defect of the dir1 mutant.

426 One cuticle-defective mutant was a knockout of MOD1, an acyl carrier protein (ACP)

427 which transports a growing FA chain between enzyme domains of FA synthase during FA

428 biosynthesis. The mod1 mutant is defective in the key FA biosynthetic enzyme enoylACP

429 reductase and has reduced levels of multiple FA species and total lipids (Lim et al., 2020).

430 Interestingly, we also found that MOD1 was > 2-fold lower in dir1 guard cells versus WT guard

431 cells after priming (Figure 3). This result supports our previous results that FA synthesis plays a

432 key role in SAR priming in guard cells (David et al., 2020). However, how DIR1 affects MOD1

433 and FA biosynthesis awaits further investigation.

435 CONCLUSION

436 Guard cells that control stomatal aperture respond to various abiotic and biotic signals

437 and have membrane-bound pattern recognition receptors that perceive bacterial pathogens. One

438 neglected area of SAR research has been the role that stomatal guard cells play in SAR. This

439 work investigates the role of SAR-related lipid transfer protein DIR1 in guard cell-specific SAR.

440 After priming and also after exposure to the bacterial pathogen Pst, stomata of WT remain at a

441 narrow aperature. In contrast, the dir1 mutant showed defects in stomatal closure. Based on the

442 multi-omics data, proteins and metabolites related to amino acid biosynthesis, secondary

443 metabolism and response to stimulus were altered in guard cells of dir1 compared to WT. For

444 example, several proteins in the methionine biosynthesis pathway and a protein related to

445 ethylene biosynthesis were decreased in the dir1 primed guard cells compared to WT. It is

446 known that ethylene is biosynthesized via methionine and ethylene plays a role in SA-regulated 
447 stomatal closure by mediating ROS and nitric oxide (Wang et al., 2020). A putative shikimate

448 dehydrogenase was also decreased in the dir1 guard cells after priming. As SA is a product of the

449 shikimate pathway and was also lower in dir1 guard cells, the slow-down in this pathway could

450 explain the decrease of stomatal closure and defense observed in the dir1 mutant during priming.

451 Our lipidomics results highlighted a role for fatty acid signaling and cuticle wax esters in the

452 primed guard cells, i.e., two 18C fatty acids as putative lipid mobile signals and two 16C wax

453 esters dependent on DIR1. These results are also correlated to a decrease in the MOD1 in the

454 dir1 guard cells. As mod1 mutants have been shown to have cuticle defects and reduced transport

455 of SA to distal tissue during SAR, this relates to the decreased SA in the dir1 guard cells. Multi-

456 omics has shown utility in discovering DIR1-dependent molecular networks in stomatal

457 immunity. The improved knowledge may facilitate effort in biotechnology and marker-based

458 breeding for enhanced plant disease resistance.

460 MATERIALS AND METHODS

$461 \quad$ Plant Growth and Bacterial Culture

A. thaliana WS seeds were obtained from Arabidopsis Biological Research Center (Ohio,

463 USA). They were suspended in deionized $\mathrm{H}_{2} \mathrm{O}$ and vernalized at $4{ }^{\circ} \mathrm{C}$ for two days before

464 planting. The seeds were cultivated in soil and grown in controlled environmental chambers in

465 short day (8-hour light/16-hour dark) environment. The temperatures during the light and dark

466 periods were $22{ }^{\circ} \mathrm{C}$ and $20^{\circ} \mathrm{C}$, respectively. Incandescent bulbs capable of emitting $140 \mu \mathrm{mol} \mathrm{m}^{-2}$

$467 \mathrm{~s}^{-1}$ at the leaf surface were used in the growth chamber with a relative humidity of about $60 \%$. A

468 dome was placed over the flat until seeds began germination. After 2 weeks of growth, seedlings 
469 were transferred into individual pots. Plants were watered weekly, kept in the chamber until

470 mature rosette (stage 3.9), and observed at 5-weeks of age.

472 induction was used for the experiments. Agar media plates were made using King's B media

473 protocol. A 1-liter solution contained $20 \mathrm{~g}$ Protease peptone No. 3, $1.5 \mathrm{~g} \mathrm{~K}_{2} \mathrm{HPO}_{4}(\mathrm{~s}), 0.75 \mathrm{~g}$

$474 \mathrm{MgSO}_{4}(\mathrm{~s}), 10 \mathrm{~mL}$ glycerol, $15 \mathrm{~g}$ agar, and deionized $\mathrm{H}_{2} \mathrm{O}$ King's B Media was autoclaved, and 475 antibiotics Rifampicin $(25 \mathrm{mg} / \mathrm{L})$ and Kanamycin $(50 \mathrm{mg} / \mathrm{L})$ were added once the solution is

476 cooled. Solution with agar was used for plates and Pst colonies were streaked on this medium

477 and incubated for overnight at $28^{\circ} \mathrm{C}$. Pst colonies were grown in the same King's B media

478 without agar in solution overnight, pelleted by centrifugation at $6000 \mathrm{x} \mathrm{g}$ for $10 \mathrm{~min}$, and used for

479 treatment of Arabidopsis plants.

\section{Stomata Aperture Measurements}

482 Primary inoculation occurred via needless syringe infiltration, where the leaves were

483 either primed with Pst DC3000 $\left(\mathrm{OD}_{600}=0.02\right)$ suspended in $10 \mathrm{mM} \mathrm{MgCl}_{2}$ or mock-treated with

$48410 \mathrm{mM} \mathrm{MgCl}_{2}$. At 3 days post inoculation, the leaf opposite to the injected leaf was detached for

485 a secondary treatment. In the secondary treatment, the leaves were either floated in $10 \mathrm{mM}$

$486 \mathrm{MgCl}_{2}$ or in Pst DC3000 $\left(\mathrm{OD}_{600}=0.2\right.$, in $\left.10 \mathrm{mM} \mathrm{MgCl}_{2}\right)$ in small petri-dishes. Three leaves were

487 used for each time point and secondary treatment group, and only one leaf was collected from

488 each plant. Stomatal apertures were measured at three time points: $0 \mathrm{~h}, 1 \mathrm{~h}$ and $3 \mathrm{~h}$. The leaves

489 were collected and peeled using clear tape. The peel from abaxial side of the leaf was then placed

490 on a microscope slide and images were collected using a DM6000B light microscope (Leica,

491 Buffalo Grove, IL USA) This experiment was repeated 3 times to image 50 stomata from each 
492 replicated treatment and a total of 150 stomata measurements from 3 independent replicates were

493 analyzed for each time point. Stomatal apertures were measured using ImageJ software (National

494 Institutes of Health, Bethesda, MD, USA, (http://imagej.nih.gov/ij/).

\section{Pst DC3000 Entry and Growth Assays}

To measure how much bacteria entered the apoplast after three hours, nine plants from

498 three independent experiments were grown to 5-weeks and prime-treated via infiltration with

499 either Pst DC3000 $\left(\mathrm{OD}_{600}=0.02\right)$ or mock-treated with $10 \mathrm{mM} \mathrm{MgCl}_{2}$. Three days later, the leaf

500 opposite to the one infected was detached and floated in Pst $\left(\mathrm{OD}_{600}=0.2\right)$ solution for both mock

501 and primed plants. After $3 \mathrm{~h}$, the leaf was placed in a Falcon tube with $0.02 \%$ Silwet (Su et al,

502 2017), vortexed for 10 seconds, dried with sterile Kim wipes, wrapped in clean aluminum foil,

503 and taken to Laminar flow hood for aseptic treatment. In the hood, an autoclaved hole-puncher

504 was used to obtain one disk from each leaf ( $0.5 \mathrm{~cm}$ diameter $)$, and the disk was placed in $100 \mu \mathrm{L}$

505 sterile $\mathrm{H}_{2} \mathrm{O}$. Each leaf disk was then ground using an autoclaved plastic grinding tip, and $10 \mu \mathrm{L}$

506 of the solution was collected to make a 1:1000 serial dilution. From the dilution, $100 \mu \mathrm{L}$ was

507 collected and plated on agar media containing Rifampicin (25 mg/L) and Kanamycin (50 mg/L).

508 After 2 days of incubation at $28^{\circ} \mathrm{C}$, the colonies on the plate were counted. The experiment was

509 done 3 times with 3 replicates each time. The bacterial counts of nine replicates were used to

510 calculate mean and standard error.

511 Pst growth experiment determines how much bacteria grow in the apoplast after 3 days.

512 Arabidopsis plants (9 independent replicates) were grown to 5-weeks and either mock or prime-

513 treated. After three days of treatment, the rosette leaves were sprayed with Pst DC3000 $\left(\mathrm{OD}_{600}=\right.$

514 0.2) and a dome was put on top for 24 hours. After 24 hours, the dome was removed, and the 
515 plants were left in growth chamber for another 48 hours. One opposite leaf of each plant was

516 then detached, washed in $0.02 \%$ Silwet, and one disk was taken from leaf to make a 1:1000 serial

517 dilution and plate it on media. Colonies were counted to determine how much bacteria were able

518 to grow in the apoplast. The experiment was repeated 3 times with 3 replicates each time. The

519 bacterial counts from the nine replicates were used to calculate mean and standard error.

521 Isolation of Enriched Guard Cells for Multi-omics Experiments

523 each sample 144 mature leaves were collected from 36 individual plants. After removing the

524 midvein with a scalpel, the leaves were blended for 1 minute in a high-speed blender with 250

$525 \mathrm{~mL}$ of deionized water and ice. The sample was then filtered through a $200 \mu \mathrm{m}$ mesh filter. This

526 process was repeated 3 times to obtain intact stomatal guard cells, which were collected

527 immediately into $15 \mathrm{~mL}$ Falcon tubes, snap frozen in liquid nitrogen, and stored in $-80{ }^{\circ} \mathrm{C}$. Guard

528 cell viability and purity was verified by staining with fluorescein diacetate and neutral red dye,

529 which showed that guard cells remained intact and viable.

\section{3-in-1 Extraction of Proteins, Metabolites and Lipids from Guard Cell Samples}

We adapted a protocol to simultaneously extract metabolites, lipids, and proteins from a

533 single whole leaf or guard cell sample (Kang et al., 2021). Briefly, a chloroform and methanol

534 solution is added to samples that are in an aqueous isopropanol solution. This process induces the

535 formation of two solvent layers - an upper aqueous phase containing hydrophilic metabolites,

536 and a lower organic phase containing lipids and other hydrophobic metabolites. The proteins are

537 at the interphase. Components were normalized from internal standards that were added during

538 the first step of extraction. Internal standards included, for proteins: 60 fmol digested bovine 
539 serum albumin (BSA) peptides per $1 \mu \mathrm{g}$ sample protein; for metabolites: $10 \mu \mathrm{L} 0.1 \mathrm{nmol} / \mu \mathrm{L}$

540 lidocaine and camphorsulfonic acid; and for lipids: $10 \mu \mathrm{L} 0.2 \mu \mathrm{g} / \mu \mathrm{L}$ deuterium labeled 15:0-

541 18:1(d7) phosphatidylethanolamine (PE) and 15:0-18:1(d7) diacylglycerol (DG). The lipid

542 extracts were dried under nitrogen gas to prevent oxidation and stored in $-80 \circ \mathrm{C}$. The lipid

543 extract was later dissolved in $1 \mathrm{~mL}$ isopropanol for LC-MS/MS analysis. Aqueous metabolites

544 were lyophilized and placed at $-80 \circ \mathrm{C}$. Aqueous metabolite pellets were later solubilized in 100

$545 \mu \mathrm{L} 0.1 \%$ formic acid for LC-MS/MS analysis. Protein components were collected by

546 precipitation in cold $80 \%$ acetone in the centrifuge tubes at $-20 \circ \mathrm{C}$ overnight. Acetone was

547 removed using glass pipettes, and the tubes with the protein samples were dried in a speedvac.

548

549 Protein Digestion and LC-MS/MS

$550 \quad$ Four biological replicates of mock and SAR primed guard cell samples from WT and

551 dir1 genotypes were prepared for proteomic experiments. Protein samples were resuspended in

$55250 \mathrm{mM}$ ammonium bicarbonate, reduced using $10 \mathrm{mM}$ dithiothreitol (DTT) at $22 \circ \mathrm{C}$ for $1 \mathrm{~h}$, and

553 alkylated with $55 \mathrm{mM}$ chloroacetamide in darkness for $1 \mathrm{~h}$. Trypsin (Promega, Fitchburg, WI)

554 was added for digestion (w/w for enzyme : sample $=1: 100)$ at $37^{\circ} \mathrm{C}$ for $16 \mathrm{~h}$. The digested

555 peptides were desalted using micro ZipTip mini-reverse phase (Millipore), and then lyophilized

556 to dryness. The peptides were resuspended in $0.1 \%$ formic acid for mass spectrometric analysis.

557 The bottom-up proteomics data acquisition was performed on an EASY-nLC 1200

558 ultraperformance liquid chromatography system (Thermo Scientific) connected to an Orbitrap

559 Exploris 480 with FAIMS Pro instrument (Thermo Scientific, San Jose, CA). The peptide

560 samples were loaded in $5 \mu \mathrm{L}$ injections to an IonOpticks Aurora $0.075 \times 250 \mathrm{~mm}, 1.6 \mu \mathrm{m} 120 \AA$

561 analytical column and column temperature was set to $50^{\circ} \mathrm{C}$ with a sonation oven. The flow rate

562 was set at $400 \mathrm{~nL} /$ minute with solvent $\mathrm{A}(0.1 \%$ formic acid in water $)$ and solvent B $(0.1 \%$ formic 
563 acid and $80 \%$ acetonitrile) as the mobile phases. Separation was conducted using the following

564 gradient: 3-19\% B in 108 min; 19-29\% B in 42 min; 29-41\% B in 30 min. The full MS1 scan

$565(\mathrm{~m} / \mathrm{z}$ 350-1200) was performed on the Orbitrap Exploris with a resolution of 120,000. The

566 FAIMS voltages were on with a FAIMS CV (V) set at -50 . The RF Lens (\%) was set to 40 and a

567 custom automatic gain control (AGC) target was set with a normalized AGC target (\%) set at

568 300. Monoisotopic precursor selection (MIPS) was enforced to filter for peptides with relaxed

569 restrictions when too few precursors are found. Peptides bearing $+2-6$ charges were selected

570 with an intensity threshold of 5e3. A custom dynamic exclusion mode was used with $60 \mathrm{~s}$

571 exclusion duration and isotopes were excluded. Data-dependent MS/MS was carried out with a 3

572 FAIMS CV loop $(-50,-65,-80)$. MS/MS orbitrap resolving power was set to 60,000 with $2 \mathrm{~m} / \mathrm{z}$

573 quadropole isolation. Top speed for data dependent acquisition within a cycle was set to $118 \mathrm{~ms}$

574 maximum injection time. The MS/MS mass tolerance was set to $10 \mathrm{ppm}$. Fragmentation of the

575 selected peptides by higher energy collision dissociation (HCD) was done at 30\% of normalized

576 collision energy and a $2(\mathrm{~m} / \mathrm{z})$ isolation window. The MS2 spectra were detected by defining first

577 mass scan range as $120 \mathrm{~m} / \mathrm{z}$ and the maximum injection time as $118 \mathrm{~ms}$.

578

579 Metabolite and Lipid Preparation and LC-MS/MS

580 The untargeted metabolomic approach used the high resolution Orbitrap Fusion Tribrid

581 mass spectrometer (Thermo Fisher Scientific, Waltham, MA, USA) with Vanquish ${ }^{\mathrm{TM}}$ UHPLC

582 liquid chromatography. An Accucore C18 $(100 \times 2.1)$ column was used for metabolites with

583 solvent A ( $0.1 \%$ formic acid in water) and solution B ( $0.1 \%$ formic acid in acetonitrile). The

584 column chamber temperature was to $55 \circ \mathrm{C}$. Pump flow rate was set to $0.45 \mathrm{~mL} / \mathrm{min}$. The LC

585 gradient was set to 0 min: $1 \%$ of solvent B (i.e., $99 \%$ of solvent A), 5 min: $1 \%$ of B, 6 min: $40 \%$

586 of B, $7.5 \mathrm{~min}: 98 \%$ of B, $8.5 \mathrm{~min}: 98 \%$ of B, 9 min: $0.1 \%$ of B, 10 min stop run. To enhance 
587 identification, an Acquire X MSn data acquisition strategy was used which employs replicate

588 injections for exhaustive sample interrogation and increases the number of compounds in the

589 sample with distinguishable fragmentation spectra for identification. Electrospray ionization

590 (ESI) was used in both positive and negative modes with a spray voltage for positive ions $(\mathrm{V})=$

5913500 and a spray voltage for negative ions $(V)=2500$. Sheath gas was set to 50, auxiliary gas

592 was set at 1 and sweep gas was set to 1 . The ion transfer tube temperature was set at $325{ }^{\circ} \mathrm{C}$ and

593 the vaporizer temperature was set at $350 \circ \mathrm{C}$. Full MS1 used the Orbitrap mass analyzer (Thermo

594 Fisher Scientific, Waltham, Massachusetts, USA) with a resolution of 120,000, scan range (m/z)

595 of 55-550, MIT of 50, AGC target of 2e5, 1 microscan, and RF lens set to 50\%. For untargeted

596 lipidomics, a Vanquish HPLC-Q Exactive Plus system was used with an Acclaim C30 column

$597(2.1 \mathrm{~mm} \times 150 \mathrm{~mm}, 3 \mu \mathrm{m})$. Solution A for lipids consisted of $0.1 \%$ formic acid, $10 \mathrm{mM}$

598 ammonium formate, and $60 \%$ acetonitrile. Solution B for lipids consisted of $0.1 \%$ formic acid,

$59910 \mathrm{mM}$ ammonium formate, and 90:10 acetonitrile: isopropyl alcohol. The column chamber

600 temperature was set to $40{ }^{\circ} \mathrm{C}$. Pump flow rate was set to $0.40 \mathrm{~mL} / \mathrm{min}$. The $\mathrm{LC}$ gradient was set

601 to 0 min: $32 \%$ of solvent B (i.e., $68 \%$ of solvent A), $1.5 \mathrm{~min}: 45 \%$ of B, 5 min: $52 \%$ of B, 8 min:

$60258 \%$ of B, 11 min: $66 \%$ of B, 14 min: $70 \%$ of B, 18 min: $75 \%$ of B, 21 min: $97 \%$ of B, 26 min:

$60332 \%$ of B, 32 min stop run. The method for Q Exactive Plus mass spectrometer included a 32-

604 min duration time, $10 \mathrm{~s}$ chromatogram peak width with full MS and ddMS2. Ion fragmentation

605 was induced by HCD, with positive and negative polarity switching and a default charge state of

606 1. Full MS1 used the Orbitrap mass analyzer with a resolution of 70,000, 1 microscan, AGC

607 target set to $1 \mathrm{e} 6$, and a scan range from 200 to $2000 \mathrm{~m} / \mathrm{z}$. The dd-MS2 scan used 1 microscan,

608 resolution of 35,000, AGC target 5e5, MIT of $46 \mathrm{~ms}$, loop count of 3, isolation window of 1.3

$609 \mathrm{~m} / \mathrm{z}$, and a scan range of 200 to $2000 \mathrm{~m} / \mathrm{z}$ for positive and negative polarity. 


\section{Data analysis for Proteins, Metabolites, and Lipids}

612 For LC-MS/MS proteomic data analysis, we used Proteome Discoverer ${ }^{\text {TM }} 2.4$ (Thermo

613 Fisher Scientific, Waltham, MA, USA) with the search engine SEQUEST algorithm to process

614 raw MS files. Spectra were searched using the TAIR10 protein database with the following

615 parameters: $10 \mathrm{ppm}$ mass tolerance for MS1 and 0.02 da as mass tolerance for MS2, two

616 maximum missed tryptic cleavage sites, a fixed modification of carbamidomethylation $(+57.021)$

617 on cysteine residues, dynamic modifications of oxidation of methionine $(+15.996)$ and

618 phosphorylation (+79.966) on tyrosine, serine, and threonine. Search results were filtered at $1 \%$

619 false discovery rate (FDR) and peptide confidence level was set for at least two unique peptides

620 per protein for protein identification. Relative protein abundance in primed and control dir1 and

621 WS guard cell samples was measured using label-free quantification in Proteome Discoverer ${ }^{\mathrm{TM}}$

6222.4 (Thermo Scientific, Bremen, Germany). Proteins identified and quantified in all 4 out of 4

623 sample replicates were used. Peptides in mock and primed samples were quantified as area under

624 the chromatogram peak. Peak areas were normalized by total protein amount. The average

625 intensity of four primed dir1 vs. four primed WS samples were compared as a ratio and two

626 criteria were used to identify significantly altered proteins: (1) increase or decrease of 2-fold

627 (primed dir1/primed WS), and (2) p-value from an unpaired Student's t-test less than 0.05. For

628 untargeted metabolomics, Compound Discover ${ }^{\mathrm{TM}} 3.0$ Software (Thermo Scientific, Bremen,

629 Germany) was used for data analyses. Raw files from four replicates of dir1 primed and four

630 replicates of WS primed guard cells were used as input. Spectra were processed by aligning

631 retention times. Detected compounds were grouped and gaps filled using the gap filling node in

632 Compound Discover that fills in missing peaks or peaks below the detection threshold for

633 subsequent statistical analysis. Peak area was refined from normalize areas while marking 
634 background compounds. Compound identification included predicting compositions, searching

635 mzCloud spectra database, and assigning compound annotations by searching ChemSpider,

636 pathway mapping to KEGG pathways and to Metabolika pathways was included for functional

637 analysis of the metabolites. The metabolites were scored by applying mzLogic and the best score

638 was kept. Peak areas were normalized by the positive and negative mode internal standards

639 (lidocaine and camphorsulfonic acid, respectively) added during sample preparation. For

640 untargeted lipidomics data analyses, Lipid Search 4.1 ${ }^{\mathrm{TM}}$ and Compound Discover ${ }^{\mathrm{TM}} 3.0$ (Thermo

641 Scientific, Bremen, Germany) were used. Raw files from three replicates of mock and three

642 replicates of primed guard cells were uploaded Lipid Search $4.1^{\mathrm{TM}}$ for annotation of lipids found

643 in all the samples. A mass list was generated for uploading to Compound Discover ${ }^{\mathrm{TM}} 3.0$

644 Software. This mass list was used for compound identification along with predicted

645 compositions, searching mzCloud spectra database, and assigning compound annotations by

646 searching ChemSpider. Peak areas were normalized by median-based normalization. For both

647 metabolomics and lipidomics, the average areas of four dir1 primed vs. four WS primed

648 metabolite samples were compared as a ratio and two criteria were used to determine

649 significantly altered metabolites or lipids: (1) increase or decrease of 2-fold (dir1 primed/WS

650 primed), and (2) p-value from an unpaired Student's t-test less than 0.05.

651

652 Accession Numbers and Data Repository Information

653 The datasets presented in this study can be found in online repositories. The names of the

654 repository/repositories and accession number(s) can be found below: All protein MS raw data

655 and search results have been deposited to the ProteomeXchange Consortium via the PRIDE

656 partner repository with the data set identifier PXD024991. All metabolite and lipid MS raw data 
657 and search results have been deposited to the MetaboLights data repository with the data set

658 identifier MTBLS2614.

660 Supplemental Data

661 The following supplemental materials are available.

662 Supplemental Table S1. Total proteins, metabolites, and lipids identified by LC-MS/MS

663 from guard cells of WT and dir1 knockout mutant under control and priming conditions.

Supplemental Figure S1. Singular enrichment analysis (SEA) for biological process using agriGO v2.0. shows pathway enrichment of proteins related to defense response,

668 amino acid biosynthesis, and carbon metabolism in guard cells.

Supplemental Figure S2. Singular enrichment analysis (SEA) for cellular components using agriGO v2.0 shows pathway enrichment of plastid and cell-wall related proteins.

\section{ACKNOWLEDGMENTS}

674 We thank Ms. Angelica Ortega and Mr. Ivan Grela their help with growth and maintenance of

675 Arabidopsis plants. This material is based upon work supported by the National Science

676 Foundation under Grant No. 1920420. 


\section{FIGURE LEGENDS}

680 Figure 1. Pathogen entry and growth differences in mock and primed dir1 mutant and wild

681 type (WT) Arabidopsis leaves. A. Images showing representative stomatal apertures in mock

682 and primed dir1 and WT Arabidopsis leaves after 0,1 , and $3 \mathrm{~h}$ after secondary exposure to Pst

683 DC3000. B. Quantitative measurements of 150 stomata from three replicate experiments.

684 Statistically significant differences were marked by a, b, and c. C. Pst DC 3000 entry results

685 obtained from nine biological replicates of primed and mock dir1 and WT plants. The data are

686 presented as average \pm standard error. D. Pst DC 3000 growth results obtained from nine

687 biological replicates of primed and mock plants. The data are presented as average \pm standard

688 error with all $\mathrm{p}$-value $<0.05$. cfu, colony forming unit

689

690 Figure 2. Differential changes of proteins and metabolites in mock and primed dir1 mutant

691 and WT guard cells. A. Biological functions proteins found in KEGG pathways that are

692 differentially abundant in WT versus dir1 primed guard cells. B. Biological functions

693 metabolites found in KEGG pathways that are differentially abundant in WT versus dir1 primed

694 guard cells.

696 Figure 3. Overview of the role of DIR1 in carbon metabolism, amino acid biosynthesis, and

697 hormone biosynthesis in guard cells during systemic defense response. Loss of DIR1 results

698 in altered abundance of proteins, metabolites, and lipids involved in carbon metabolism, amino

${ }^{1}$ This material is based upon work supported by the National Science Foundation under Grant No. 1920420.

${ }^{2}$ Author for contact: schen@ufl.edu

${ }^{3}$ Senior author.

The author responsible for distribution of materials integral to the findings presented in this article in accordance with the policy described in the Instructions for Authors

(www.plantphysiol.org) is: Sixue Chen (schen@ufl.edu).

S.C., and L.D. conceived and designed the research; L.D., J.K., J.N., and C.D. carried out all experimental work; L.D conducted data analysis, and L.D. and S.C. prepared manuscript. 
699 acid biosynthesis, biosynthesis of plant hormones and secondary metabolites. Proteins that were

700 decreased in dir1 guard cells in the carbon metabolism metabolic pathway included: FBA3, TIM,

701 TPI, LOS2, PKP $\alpha$, and ACO2. Proteins that were decreased in dir1 guard cells in the amino acid

702 biosynthesis metabolic pathways included: AGD2, CYSC1, CBL, MS2, MTO1, AT4G17830,

703 and AQI, and decreased metabolites in these pathways included: gly-leu, niacin, acetyl-leucine,

704 desaminotyrosine, and pyroglutamic acid. One increased metabolite in dir1 guard cells in the

705 arginine biosynthesis pathway was aminolevulinic acid. Proteins that were decreased in dir1

706 guard cells in the biosynthesis of hormones and secondary metabolites metabolic pathways

707 included: MEE32 and MOD1, and decreased metabolites and lipids in these pathways included

708 salicylic acid, stearic acid (FA 18:0), behenic acid (FA 18:1), cetyl oleate (WE 16:0/18:1) and

709 ethyl myristate (WE 16:0). One protein, EFE, and one lipid, FAO2 18:1, were increased in these

710 pathways in the dir1 primed guard cells versus WT primed guard cells. Please refer to

711 Supplemental Table 1 for abbreviations.

713 Figure 4. Differentially abundant lipids identified in dir1 and WT guard cells. A. Bar graphs

714 showing decreases of two long-chain fatty acids, stearic acid (FA 18:0) and behenic acid (FA

715 18:1) and two wax esters, cetyl oleate (WE 16:0/18:1) and ethyl myristate (WE 16:0) decreased

$716>2$-fold in the dir1 versus WT guard cells. Chemical structures of stearic acid (FA 18:0),

717 behenic acid (FA 18:1), cetyl oleate (WE 16:0/18:1) and ethyl myristate (WE 16:0) are shown.

718 The error bar represents standard deviation of the mean value.

720 Figure 5. Identification of potential interacting proteins with DIR1. A. Protein interaction

721 image was generated using Interaction Viewer at bar.utoronto.ca/eplant. Border color indicates 
722 protein location. Green lines indicate protein and DNA interactions that have been

723 experimentally determined. B. GeneMANIA tool from bar.utoronto.ca/eplant was used to predict

724 other genes/gene products associated with DIR1 (AT5G48485). Predicted, co-expression, and

725 genetic interaction networks found associated genes/gene products. Proteins identified in guard

726 cell samples are circled. Circle colors indicate increased (red), decreased (green), or unchanged

727 (blue) proteins in dir1 versus WT primed guard cells.

728

729 
bioRxiv preprint doi: https://doi.org/10.1101/2021.06.02.446770; this version posted June 2, 2021. The copyright holder for this preprint (which was not certified by peer review) is the author/funder, who has granted bioRxiv a license to display the preprint in perpetuity. It is made available under aCC-BY-NC-ND 4.0 International license.

\section{Parsed Citations}

Abdel-Ghany, SE (2009) Contribution of plastocyanin isoforms to photosynthesis and copper homeostasis in Arabidopsis thaliana grown at different copper regimes. Planta 229: 767-779

Google Scholar: Author Only Title Only Author and Title

Ádám AL, Nagy ZÁ Kátay G, Mergenthaler E, Viczián O (2018) Signals of systemic immunity in plants: Progress and open questions. Int J Mol Sci 19: 1146-1166

Google Scholar: Author Only Title Only Author and Title

Bogdanov, I. V., Shenkarev, Z O., Finkina, E. l., Melnikova, D. N., Rumynskiy, E. I., Arseniev, A S., \& Ovchinnikova, T. V. (2016) Anovel lipid transfer protein from the pea Pisum sativum: isolation, recombinant expression, solution structure, antifungal activity, lipid binding, and allergenic properties. BMC Plant Biol, 16: 107

Google Scholar: Author Only Title Only Author and Title

Cornish K, Zeevaart JA(1985) Movement of Abscisic Acid into the Apoplast in Response to Water Stress in Xanthium strumarium L. Plant Physiol 78: 623-626

Google Scholar: Author Only Title Only Author and Title

David L, Harmon AC, Chen S (2019) Plant immune responses - from guard cells and local responses to systemic defense against bacterial pathogens. Plant Signal Behav 14: e1588667

Google Scholar: Author Only Title Only Author and Title

David L, Kang J, Dufresne D, Zhu D, Chen S (2020) Multi-omics revealed molecular mechanisms underlying guard cell systemic acquired resistance. Int J Mol Sci. 27: 191-212

Google Scholar: Author Only Title Only Author and Title

David L, Kang J, Chen S (2021) Untargeted metabolomics of Arabidopsis stomatal immunity. Methods Mol Biol 2200: 413-424

Google Scholar: Author Only Title Only Author and Title

Du Z, Zhou X, Ling Y, Zhang Z, Su Z (2010) agriGO: a GO analysis toolkit for the agricultural community. Nucleic Acids Res 38(Web

Server issue): W64-70

Google Scholar: Author Only Title Only Author and Title

Fahlberg P, Buhot N, Johansson ON, Andersson MX (2019) Involvement of lipid transfer proteins in resistance against a non-host powdery mildew in Arabidopsis thaliana. Mol Plant Pathol. 20: 69-77

Google Scholar: Author Only Title Only Author and Title

Junková P, Neubergerová M, Kalachova T, Valentová O, Janda M (2021) Regulation of the microsomal proteome by salicylic acid and deficiency of phosphatidylinositol-4-kinases $\beta 1$ and $\beta 2$ in Arabidopsis thaliana. Proteomics 21: e2000223.

Google Scholar: Author Only Title Only Author and Title

Kang J, David L, Cang J, Chen S (2021) Three-in-one simultaneous extraction of proteins, metabolites and lipids for multi-omics. Front Genet 12: 635971

Google Scholar: Author Only Title Only Author and Title

He M, Ding NZ (2020) Plant unsaturated fatty acids: Multiple roles in stress response. Front Plant Sci 11: 562785

Google Scholar: Author Only Title Only Author and Title

Hirano SS, Upper CD (2000) Bacteria in the leaf ecosystem with emphasis on Pseudomonas syringae-a pathogen, ice nucleus, and epiphyte. Microbiol Mol Bio Rev 64 :624-653

Google Scholar: Author Only Title Only Author and Title

Lascombe MB, Bakan B, Buhot N, Marion D, Blein JP, Larue V, Lamb C, Prangé T (2008) The structure of "defective in induced resistance" protein of Arabidopsis thaliana, DIR1, reveals a new type of lipid transfer protein. Protein Sci 17: 1522-1530

Google Scholar: Author Only Title Only Author and Title

Li F, Wu X, Lam P, Bird D, Zheng H, Samuels L, Jetter R, Kunst L (2008) Identification of the wax ester synthase/acyl-coenzyme A: diacylglycerol acyltransferase WSD1 required for stem wax ester biosynthesis in Arabidopsis. Plant Physiol 148: 97-107

Google Scholar: Author Only Title Only Author and Title

Lim GH, Shine MB, de Lorenzo L, Yu K, Cui W, Navarre D, Hunt AG, Lee JY, Kachroo A, Kachroo P (2016) Plasmodesmata localizing proteins regulate transport and signaling during systemic acquired immunity in plants. Cell Host Microbe 19: 541-549

Google Scholar: Author Only Title Only Author and Title

Lim GH, Liu H, Yu K, Liu R, Shine MB, Fernandez J, Burch-Smith T, Mobley JK, McLetchie N, Kachroo A, Kachroo P (2020) The plant cuticle regulates apoplastic transport of salicylic acid during systemic acquired resistance. Sci Adv 6: eaaz0478

Google Scholar: Author Only Title Only Author and Title

Maldonado AM, Doerner P, Dixon RA, Lamb CJ, Cameron RK (2002) A putative lipid transfer protein involved in systemic resistance signaling in Arabidopsis. Nature 419: 399-403

Google Scholar: Author Only Title Only Author and Title 
bioRxiv preprint doi: https://doi.org/10.1101/2021.06.02.446770; this version posted June 2, 2021. The copyright holder for this preprint (which was not certified by peer review) is the author/funder, who has granted bioRxiv a license to display the preprint in perpetuity. It is made available under aCC-BY-NC-ND 4.0 International license.

Mallikarjun V, Clarke DJ, Campbell CJ (2012) Cellular redox potential and the biomolecular electrochemical series: a systems

hypothesis. Free Radic Biol Med. 53: 280-288

Google Scholar: Author Only Title Only Author and Title

Martinière A, Gibrat R, Sentenac H, Dumont X, Gaillard I, Paris N (2018) Uncovering pH at both sides of the root plasma membrane interface using noninvasive imaging. Proc Natl Acad Sci 115: 6488-6493

Google Scholar: Author Only Title Only Author and Title

Melotto M, Underwood W, He SY (2008) Role of stomata in plant innate immunity and foliar bacterial diseases. Ann Rev Phytopathol 46: 101-122

Google Scholar: Author Only Title Only Author and Title

Misra BB, Chaturvedi R (2015) When plants brace for the emerging pathogens. Physiol Mol Plant Path. 92: 181-185

Google Scholar: Author Only Title Only Author and Title

Nguyen C, Haushalter RW, Lee DJ, Markwick PR, Bruegger J, Caldara-Festin G, Finzel K, Jackson DR, Ishikawa F, O'Dowd B, McCammon JA, Opella SJ, Tsai SC, Burkart MD (2014) Trapping the dynamic acyl carrier protein in fatty acid biosynthesis. Nature 505:

427-431

Google Scholar: Author Only Title Only Author and Title

Pang Q, Zhang T, Lin C, Kong W, Chen S. (2020) Proteomics and phosphoproteomics revealed molecular networks of stomatal immune responses. Planta 252: 66

Google Scholar: Author Only Title Only Author and Title

Rebaque D, Del Hierro I, López G, Bacete L, Vilaplana F, Dallabernardina P, Pfrengle F, Jordá L, Sánchez-Vallet A, Pérez R, Brunner F, Molina A, Mélida H (2021) Cell wall-derived mixed-linked $\beta-1,3 / 1,4-g l u c a n s$ trigger immune responses and disease resistance in plants.

Plant J 106 :601-615

Google Scholar: Author Only Title Only Author and Title

Saint-Vincent PM, Ridout M, Engle NL, Lawrence TJ, Yeary ML, Tschaplinski TJ, Newcombe G, Pelletier DA(2020) Isolation, characterization, and pathogenicity of two Pseudomonas syringae pathovars from Populus trichocarpa seeds. Microorganisms 8: 11371156

Google Scholar: Author Only Title Only Author and Title

Santelia D, Lawson T (2016) Rethinking guard cell metabolism. Plant Physiol 172:1371-1392

Google Scholar: Author Only Title Only Author and Title

Shah J, Zeier J (2013) Long-distance communication and signal amplification in systemic acquired resistance. Front. Plant Sci. 4:1-16 Google Scholar: Author Only Title Only Author and Title

Sels J, Mathys J, De Coninck BM, Cammue BP, De Bolle MF (2008) Plant pathogenesis-related (PR) proteins: a focus on PR peptides.

Plant Physiol Biochem 46: 941-50

Google Scholar: Author Only Title Only Author and Title

Su J, Zhang M, Zhang L, Sun T, Liu Y, Lukowitz W, Xu J, Zhang S (2017) Regulation of stomatal immunity by interdependent functions of a pathogen-responsive MPK3/MPK6 cascade and abscisic acid. Plant Cell 29: 526-542

Google Scholar: Author Only Title Only Author and Title

Wang HQ, Sun LP, Wang LX, Fang XW, Li ZQ, Zhang FF, Hu X, Qi C, He JM (2020) Ethylene mediates salicylic-acid-induced stomatal closure by controlling reactive oxygen species and nitric oxide production in Arabidopsis. Plant Sci 294:110464

Google Scholar: Author Only Title Only Author and Title

Weigel M, Varotto C, Pesaresi P, Finazzi G, Rappaport F, Salamini F, Leister D. (2003) Plastocyanin is indispensable for photosynthetic electron flow in Arabidopsis thaliana. J Biol Chem 278: 31286-9

Google Scholar: Author Only Title Only Author and Title

Wittek F, Hoffmann T, Kanawati B, Bichlmeier M, Knappe C, Wenig M, Schmitt-Kopplin P, Parker JE, Schwab W, Vot AC (2014) Arabidopsis ENHANCED DISEASE SUSCEPTIBILITY1 promotes systemic acquired resistance via azelaic acid and its precursor 9-oxo nonanoic acid. J Exp Bot 65: 5919-5931

Google Scholar: Author Only Title Only Author and Title

Wong LH, Gatta AT, Levine TP (2019) Lipid transfer proteins: the lipid commute via shuttles, bridges and tubes. Nat Rev Mol Cell Biol $20: 85-101$

Google Scholar: Author Only Title Only Author and Title

Yu K, Soares JM, Mandal MK, Wang C, Chanda B, Gifford AN, Fowler JS, Navarre D, Kachroo A, Kachroo P (2013) A feedback regulatory loop between G3P and lipid transfer proteins DIR1 and AZ1 mediates azelaic-acid-induced systemic immunity. Cell Rep. 3:1266-1278

Google Scholar: Author Only Title Only Author and Title

Zhu M, Jeon BW, Geng S, Yu Y, Balmant K, Chen S, Assmann SM (2016) Preparation of epidermal peels and guard cell protoplasts for cellular, electrophysiological, and -omics assays of guard cell function. Methods Mol Biol 1363: 89-121

Google Scholar: Author Only Title Only Author and Title 
bioRxiv preprint doi: https://doi.org/10.1101/2021.06.02.446770; this version posted June 2, 2021. The copyright holder for this preprint (which was not certified by peer review) is the author/funder, who has granted bioRxiv a license to display the preprint in perpetuity. It is made
available under aCC-BY-NC-ND 4.0 International license.

Zhu M, Dai S, Chen S (2012) The stomata frontline of plant interaction with the environment-perspectives from hormone regulation. Front Biol 7: 96-112

Google Scholar: Author Only Title Only Author and Title 